\title{
A Numerical Waveform Design Approach to Decorrelate Target and Noise
}

\author{
Robert J. Bonneau, Michael C. Wicks \\ Air Force Research Lab \\ 26 Electronics Pkwy \\ Radar Signal Processing Branch \\ Rome, NY 13441
}

\begin{abstract}
Traditional radar detection relies on predefined models for assessment of probability of detection and probability of false alarm. These models assume a target uncorrelated with clutter or other sources of interference. This assumption concerning decorrelation between the target and interference is not strictly true. We propose an iterative approach to design "optimal" waveforms that maximally decorrelate target and clutter returns while still keeping target signal-tointerference plus noise ratios (SINR) levels maximized.
\end{abstract}

\section{INTRODUCTION}

An often sought after goal in radar is to maximize the receiver operating characteristic curve or ROC for any given target in a clutter plus noise interference environment[3]. Thus we simultaneously want low probability of false alarm and high probability of detection. To achieve low probability of false alarm, there must be little if any correlation between a target and the background, otherwise, classical filters remain unable to separate desired target returns from interference. Background clutter in this case includes other targets resembling the threat target of interest. Although our method is designed for binary decisions it can be extended to multiple hypothesis testing. Additionally we must . keep the target response for a given radiated waveform high in order to achieve adequate signal to noise. We thus develop a numerical waveform design approach to meet these criteria, and show improved detection performance for a target and clutter example.

\section{W AVEFORM INTERACTION}

We begin by defining a linear FM or 'chirp' signal with sufficient bandwidth to adequately resolve (over-resolve) the threat target under surveillance. If we denote a unit pulse function $\tilde{u}(t)=1$ for , else
0 , and a modulated waveform $f(t)$, with carrier frequency $\omega_{0}$, then the waveform can be mathematically described as

$$
f(t)=\tilde{u}(t) e^{j b t^{2}} e^{j 2 \pi \omega}
$$

We denote $b$ as the chirp slope describing the rate over which the modulated waveform varies in angular frequen cy between $\omega_{1}<\omega<\omega_{2}$. If we limit the chirp signal to duration $T$, with $-T / 2 \leq t \leq T / 2$, then the bandwidth of the chirp signal is

$$
B_{\mathrm{c}}=b T
$$

We now denote our target to have an impulse response $T(t)$ with our received signal $r_{T}(t)$ equal to

$$
r_{T}(t)=f(t) \otimes T(t)
$$

After our return signal is correlated with the transmit signal we have.

$$
\hat{r}_{T}(t)=f(t) \circ r(t)
$$

where $\circ$ indicates correlation operation. Similarly for our clutter impulse response, $C(t)$, then our received signal is

$$
r_{c}(t)=f(t) \otimes C(t)
$$

and after correlation

$$
\hat{r}_{C}(t)=f(t) \circ r(t)
$$

\section{W AVEFORM CONSTRAINS}

Next we must impose constraints on the transmitted waveform such that the target 
response is decorrelated from the clutter response, with the target return large enough to meet or exceed some prior Signal -to-Noise Ratio (SNR) constraint [1]. To des cribe the correlation between target and clutter we have the test metric

$$
m_{r}=\hat{r}_{C} \circ \hat{r}_{T}
$$

For joint optimization of the target to clutter decorrelation over observation interval as a function of bandwidth, we wish to find the frequency range $B_{c}$ for any given center frequency $\omega$, such that

$$
B_{c} \Rightarrow \min \left(m_{r}\right) \text { given that } \max \left(\hat{r}_{T}\right)
$$

\section{W AVEFORM ITERATIVE SEARCH PROCEDURE}

Since for each target we wish to minimize the correlation test metric while maximizing the returned target signal, but the absolute min and max for each metric will not usually be simultaneously attainable, since the correlation metric is a function of the target response itself. We thus initialize our process by setting a threshold for the maximum $\hat{r}_{T}$ necessary to achieve the desired SNR [1] and then find the point at which $m_{r}$ is minimized after $\hat{r}_{T}$ has surpassed a pre-specified threshold . To minimize $m_{r}$, we apply a gradient numerical search technique by specifying a center frequency $\omega_{o}$ for each initial condition to first minimize $m_{r}$ given that we are within the threshold constraint for $\hat{r}_{T}$. Using the Newton method [4] of numerical approximation we first approximate the numerical derivative of $\hat{r}_{T}$ using

$$
m^{\prime}\left(B_{k}\right)=\frac{d m_{r}}{d B} \approx \frac{m_{r}\left(B_{k}\right)-m_{r}\left(B_{k-1}\right)}{B_{k}-B_{k-1}}
$$

where the subscripts $\mathrm{k}$ and $\mathrm{k}-1$ refer to the succession of $B$ values used to find the correlation metric minimum. Our method for minimization of the correlation metric is then found using the iterative formula

$$
B_{k+1}=B_{k}-\frac{m\left(B_{k}\right)}{m^{\prime}\left(B_{k}\right)}
$$

Inserting equation 8 into 9 we have

$$
B_{k+1}=B_{k}-\frac{m_{r}\left(B_{k}\right)\left(B_{k}-B_{k-1}\right)}{m_{r}\left(B_{k}\right)-m_{r}\left(B_{k-1}\right)}
$$

Thus we iterate the above equation until we find a minima given that $\hat{r}_{T}$ is greater that threshold $T_{r}$

$$
\hat{r}_{T}>T_{r}
$$

If these criteria are not met then we change to a new center frequency $\omega$ and repeat the process. The bandwidth associated with the optimal target characteristics we will denote $B_{o p t}$ at center frequency $\omega_{c}$.

\section{DEMONSTRATION OF DETECTION PERFORMANCE}

We demonstrate the difference in detection performance using the Weiner matched filter detection methodology. Let $s$ be the complex vector of target reflectivity, $\underline{\underline{n}}$ a noise vector (including clutter effects) and $\underline{x}$ equals the combined signal vector.

$$
\underline{x}=\underline{s}+\underline{n}
$$

We now define the expected value of the $x$ under the null hypothesis $\mathrm{H}_{0}$, target absent, to be 0 , and under the alternative hypothesis $\mathrm{H}_{1}$, target present, to be $\mathbf{s}$, the complex reflectivity of the target. Additionally, we define the covariance of the noise/clutter vector as $M$, we have

$$
\begin{gathered}
E\left\{x \mid H_{0}\right\}=0 \quad E\left\{x \mid H_{1}\right\}=s \\
E\left\{n n^{h} \mid H_{i}\right\}=M
\end{gathered}
$$

We thus define the matched filter detection test statistic given signal and noise estimates as follows:

$$
|y|=\frac{\left|s^{h} M^{-1} x\right|}{\sqrt{s^{h} M^{-1} x}}>\mu \Leftrightarrow H_{0}
$$

A target is judged to be present if the test statistic $|y|$ is greater than threshold $\boldsymbol{\mu}$ and absent if less 
than the threshold. We then compute the probability of detection $B$ and probability of false alarm $P_{F A}$ for a fixed threshold $\mu$ with

$$
\begin{aligned}
& P_{D}=\frac{\# t \arg e t s \_ \text {detected } \mid t \arg e t}{\# t \arg e t s}=\frac{n_{t g t}}{N_{\text {tgt }}} \\
& P_{F A}=\frac{\# t \arg \text { ets_detected } \mid \text { clutter }}{\# t \text { arg ets }}=\frac{n_{f a}}{N_{t g t}}
\end{aligned}
$$

Results show improved performance using a matched waveform selection methodology over an arbitrary waveform selection methodology for a given class of targets under the following conditions. Figures $1 \mathrm{a}$ and $1 \mathrm{~b}$ demonstrates results using simulated test targets exhibiting a dominant impulse response with a variety of minor peaks. Without adaptive waveform selection the probability of false alarm remains quite high over a wide variation in SNR. This is due to our lack of ability to distinguish between two targets with a poorly matched waveform. After adaptive waveform selection, the probability of detection remains high while our probability of false alarm is significantly reduced.

\section{Conclusion}

Our numerical search technique for waveform design allows us to find the optimal chirp function to decorrelate target returns from interference while maintaining adequate detection performance, even for low SNR. This methodology can be extended to other classes of waveforms covering a much wider class of threat targets. This technique promises to open new approaches to target matched waveform design and allow for the detection and identification of targets under more stressing clutter and interference conditions.
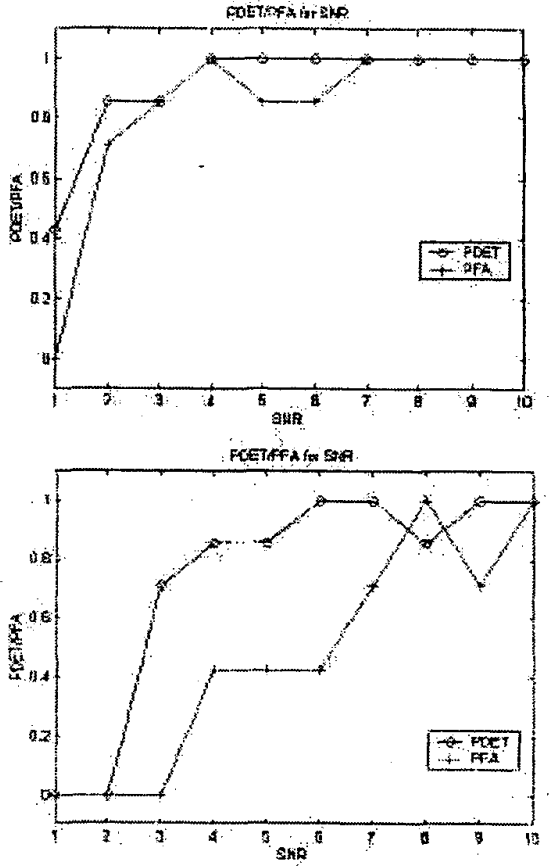

Fig. 1a, lb Comparison of detection performance without and with adaptive waveform selection.

\section{REFERENCES}

[1] Pillai, S.U., Oh, H.S., Youla, D.C., Guerci, J.R., "Optimum Transmit-Receiver Design, in the Presence of Signal-Dependent Interference and Channel Noise", IEEE Transactions on Information Theory, Vol. 46, No. 2, March 2000.

[2] Skolnik, M.I., Introduction to Radar Systems, McGraw Hill, New York, 1980.

[3] VanTrees, H.L, Detection, Estimation and Modulation Theory, Vol. 1, Wiley, New York, 1968.

[4] Widrow, B., Stearns S., Adaptive Signal Processing, Prentice Hall, Englewood Cliffs, N.J.. 1985. 